\title{
Proposing a Patellar Tendinopathy Screening tool following a systematic review
}

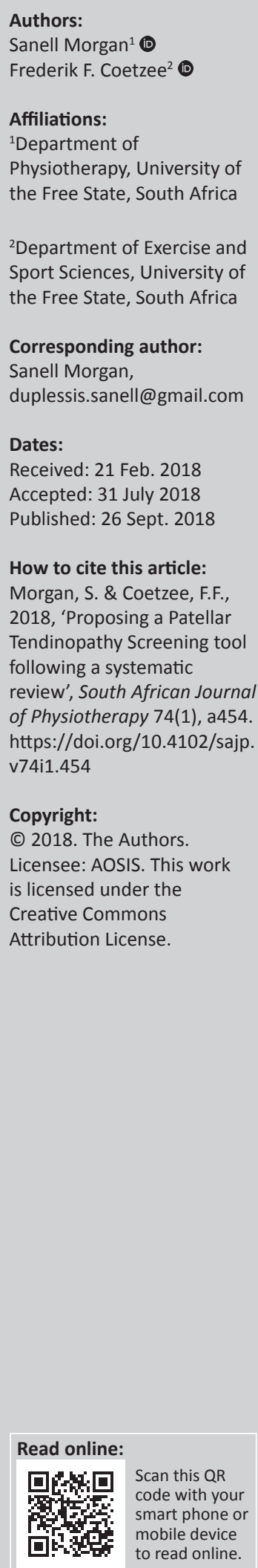

Background: Patellar tendinopathy (PT) is an overuse injury of the knee. The mechanism of injury is associated with repetitive stress on the patellar tendon of the knee as a result of explosive movement. Patellar tendinopathy is prevalent in all populations and is associated with intrinsic and extrinsic risk factors.

Objectives: Primarily, the objective was to report on the intrinsic and extrinsic risk factors for PT, entailing a systematic review of the literature; the secondary objective was to use these risk factors to compile a proposed PT screening tool from the review and standard outcome measures.

Method: A systematic review was undertaken according to the Preferred Reporting Items for Systematic Reviews and Meta-Analyses (PRISMA) guidelines. Elimination criteria of the articles included duplicates, titles, abstracts and methodological quality. The evidence was collected, characterised with regard to the intrinsic and extrinsic risk factors and summarised descriptively.

Results: The search yielded 157 feasible articles prior to commencement of article elimination. Six articles were included with a mean methodological quality score of $69 \%$. Eight intrinsic and five extrinsic risk factors were identified. These identified risk factors are all relevant to the pathology and formed the basis for a proposed PT screening tool. The Victorian Institute of Sports Assessment for Patellar Tendinopathy Questionnaire, Visual Analog Scale and the Pain Provocation Test are also included in the proposed test.

Conclusion: Intrinsic and extrinsic risk factors for PT were identified, and consequently, the proposed PT screening tool was formulated for possible future testing in appropriate studies.

Clinical implications: Prevention of PT through intrinsic and extrinsic risk factor identification, and implementation in the clinical setup as a possible outcome measurement tool with which to verify functional improvement in PT rehabilitation.

\section{Introduction}

Patellar tendinopathy (PT), an overuse injury (Reinking 2016) often referred to as 'jumper's knee', commonly occurs in sports that particularly involve jumping. The mechanism of injury is associated with continuous and repetitive stress on the patellar tendon of the knee (Celebi et al. 2016) resulting from explosive movement (Van Ark et al. 2016). This pathology occurs in all populations (Celebi et al. 2016), with an estimated prevalence of $8.5 \%$ in recreational athletes who usually have a less intense training regimen (Vetrano et al. 2013). However, in elite athletes the prevalence of PT is as high as 13\%-20\% (Stuhlman et al. 2016) and can even reach a percentage of $40 \%$ in elite athletes involved in sports with a high demand (Abat \& Sanchez-Ibañez 2014) on the extensor mechanism of the knee (Steunebrink et al. 2013). The main causes are intrinsic (Malliaras \& O'Neill 2017) and extrinsic risk factors (Hägglund, Zwerver \& Ekstrand 2011; Reinking 2016).

The physical diagnosis of PT is based on clinical and predominantly ultrasound examination, although findings may not necessarily be associated with the severity of the symptoms (Celebi et al. 2016). The rehabilitation of PT is typically non-operative and requires an evidence-based approach founded on the research literature. This is enhanced by the clinical judgement of the rehabilitation team, their understanding of the rehabilitation intervention and wise implementation thereof (Rutland et al. 2010), whilst still keeping the athlete's return to sport goals in mind 
(Reinking 2016). Patellar tendinopathy rehabilitation is an encompassing management of the athlete with the fundamental focus on exercise (Van Ark et al. 2016). However, with the possibility of enduring persistent symptoms, full recovery from this pathology is not always certain (De Vries et al. 2017).

Even though PT is a familiar pathology amongst elite and recreational athlete populations (Reinking 2016), there is modest evidence for risk screening tools in PT assessment and rehabilitation. A necessity is that the rehabilitation team's clinical reasoning and progress of the rehabilitation process must be founded on authenticated subjective and objective measurements (Reinking 2016). As a result of the unconvincing (Peters et al. 2016) and challenging track record of PT rehabilitation (Rodriguez-Merchan 2013; Van Der Worp et al. 2011b), compelling and trustworthy screening tools are important for routine clinical practice. These should be used as outcomes to validate the efficiency of the treatment, monitor progress and identify regression of the pathology (Celebi et al. 2016).

Prior systematic reviews have identified a list of risk factors for PT, but there is a paucity of information on PT screening tools for practical implementation in the clinical setup. Therefore, a need was identified for an in-depth systematic literature review to form the basis of a draft screening tool for PT as well as updating current data in this field. Such a PT screening tool may facilitate PT research, once it has been appropriately developed. Clinically, it may then assist in prevention of PT by early identification of risk factors and serve as an outcome measure in rehabilitation. Thus, the primary objective of this study was to systematically identify and appraise studies reporting on the intrinsic and extrinsic risk factors for $\mathrm{PT}$, with the secondary aim of using the identified risk factors in a proposed PT screening tool, in combination with appropriate outcome measures.

\section{Method}

Systematic reviews are a cornerstone in research for complete, accurate and reliable recapitulating of evidence, and consequently provide several benefits to clinicians (Liberati et al. 2009). This systematic review was undertaken according to the Preferred Reporting Items for Systematic Reviews and Meta-Analyses (PRISMA) guidelines (Moher et al. 2009). The inclusion and exclusion criteria in Box 1 were used as a basis for the consideration of articles.

\section{Search strategy}

The authors conducted two independent systematic review searches, first in October 2017 and in January 2018, for articles published between January 2010 and September 2017, with an allotted time period (approximately three months apart) between the searches by the authors. This ensured that results cross-referenced and that all eligible articles were included in the review. This interval was chosen to be a supplementary addition to a previously published systematic review on the
BOX 1: Inclusion and exclusion criteria.

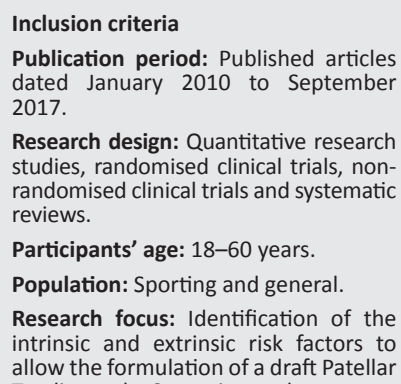

causative risk factors and rehabilitation for PT in which articles were selected only up to October 2015 (Morgan, Janse van Vuuren \& Coetzee 2016). Clinical Sports Medicine (5th edition) by Brukner and Khan (2017) was used as a basis to identify the words for the Boolean phrases.

EBSCO host electronic databases were searched and included the following: Africa-Wide Information, ERIC, Academic Search Complete, AHFS Consumer Medication Information, CINAHL with Full Text, MEDLINE with Full Text, Health Source: Nursing/Academic Edition, Health Source Consumer Edition, PsycARTICLES, Humanities Source, PsycEXTRA, SocINDEX with Full Text, PsycINFO, PsycTESTS, and SPORTDiscus with Full Text.

The following keywords were applied in the search strategy for the identification of applicable articles for the review:

('patella* tendinopath*' or [patella* and tendinit*])('intrinsic factor*' or age or gender or 'body composition*' or 'fat mass' or 'body weight' or 'body mass index' or injur* or 'joint instability*' or 'musc* strength' or 'musc* power' or 'range of motion' or 'range of movement' or 'anatomic* alignment*' or 'postural stability*' or 'sport* specific technique*' or 'level of skill'" or 'skill* level*' or 'extrinsic factor*' or strapping or bracing or 'foot wear' or footwear* or shoe* or 'training surface*' or 'eccentric decline squat*' or 'skill* acquisition' or proprioception* or flexib* or 'muscle activat*' or etiolog* or aetiolog*) AND (rehab* or 'return to sport' or 'return to play' or 'motor re-educat*') and (exercise* or train* or sport*)

\section{Study selection}

Each author undertook the study selection process according to the inclusion and exclusion criteria in Box 1. All uncertainties regarding the eligibility of articles were discussed by the authors to clarify and agree upon any initial discrepancy. Figure 1 demonstrates the methodology of the search strategy in determining the final set of articles for evaluation.

\section{Methodological quality of the articles included in the review}

The eligibility and quality of the articles were appraised by the authors using two checklists (Table 1). More than one checklist was needed because of the dissimilar research 
designs described in the different articles. These specific checklists were selected to assist in defining the methodological quality of each article because of their good validity and reliability in the literature (National Institute for Health and Excellence [NICE] 2012; Shea et al. 2009; Winn 2013). The checklists included the AMSTAR (Shea et al. 2009) and NICE (2012) Methods for the Development of NICE Public Health Guidance checklists. The individual methodology scoring for each article is displayed in Appendix 1.

The methodological quality scoring was performed on 13 articles that met the inclusion criteria, with 6 articles remaining for the systematic review.

\section{Data extraction}

The data contained in the eligible articles were extracted and incorporated into a customised Microsoft Excel data spreadsheet developed by the authors (Tables 2 and 3). The documented information included authors, year of publication, aim of the study, type of study, the population, level of participation and intrinsic and extrinsic risk factors defined in each article. The intrinsic and extrinsic risk factors

\begin{tabular}{|c|c|}
\hline $\begin{array}{l}\text { Eligible Articles } \\
\qquad(n=157)\end{array}$ & - Check for duplicates resulted in 34 exclusions \\
\hline $\begin{array}{l}\text { Eligible Articles } \\
\qquad(n=123)\end{array}$ & - Screening of titles resulted in 49 exclusions \\
\hline $\begin{array}{l}\text { Eligible Articles } \\
\qquad(n=74)\end{array}$ & - Screening of abstracts resulted in 21 exclusions \\
\hline $\begin{array}{l}\text { Eligible Articles } \\
\qquad(n=53)\end{array}$ & $\begin{array}{l}\text { - Screening of full texts resulted in } 40 \text { exclusions } \\
\text { (excluded because of no intrinsic and extrinsic risks } \\
\text { factors for PT, age of participants, other tendinopathy } \\
\text { pathologies and qualitative research designs) }\end{array}$ \\
\hline $\begin{array}{l}\text { Eligible Articles } \\
\qquad(n=13)\end{array}$ & $\begin{array}{l}\text { - Screening based on methodology quality resulted } \\
\text { in } 7 \text { exclusions }\end{array}$ \\
\hline & Eligible Articles ( $n=6)$ \\
\hline
\end{tabular}

PT, Patellar tendinopathy.

FIGURE 1: Flow diagram of search strategy to determine the final sample of articles for the systematic review. were identified in the results and discussion of each article during the data extraction process. The authors conducted the data extraction individually, which was followed by a final mutual check.

\section{Analysis of data}

Combining of the data for the formulation of a meta-analysis was not the intention of this systematic review because of the differences of the results in terms of the variety of articles with different study populations. All the empirical evidence was collected, characterised with regard to the intrinsic and extrinsic risk factors for PT and summarised descriptively.

\section{Ethical considerations}

Ethics approval was obtained from the Ethics Committee of the Faculty of Health Sciences, University of the Free State (approval no. 181/2015).

\section{Results}

The collective results of both independent searches yielded 157 feasible articles for inclusion prior to the commencement of article elimination (Figure 1 and Table 1). Six articles met all the inclusion criteria and are shown in Table 2. These were included in the systematic review with a mean methodological quality score of $69 \%$ (Table 1). Only quantitative research designs and systematic reviews formed part of the included articles with, surprisingly, no randomised clinical trials. This systematic review led to the identification of eight intrinsic and five extrinsic risk factors, with the results of these articles being discussed.

The demographic information obtained from the systematic review shows that all six of the included articles described the study population. Two articles consisted of exclusively either male or female participants, whilst the other four articles described both male and female participants. Eighty-three per cent $(n=5)$ of the included articles provided detail on the level

TABLE 1: Methodological assessment of included articles.

\begin{tabular}{|c|c|c|c|c|}
\hline Study design & Methodological checklist & Range of quality scoring & Quality scoring average & Article exclusion reason \\
\hline Systematic reviews & AMSTAR checklist & $8 / 11(n=1)$ & $73 \%(n=1)$ & Methodology scoring of $50 \%$ or less \\
\hline Quantitative research & National Institute for Health and Excellence checklist & $15 / 27-22 / 27(n=5)$ & $67 \%(n=6)$ & Methodology scoring of $50 \%$ or less \\
\hline
\end{tabular}

TABLE 2: Characteristics of the included articles.

Authors and year of publication Aim of the study

De Vries et al. (2015a) Investigating if the identified risk factors in a previous study in 2008 can prospectively be identified as risk factors of PT in 2011

Mendonça et al. (2016) Determine the connotation of lower limb muscle strength and lower limb alignment, range of motion or flexibility in male athletes with PT

Van Der Worp et al. (2011b)

Toppi et al. (2015)

Identification of the risk factors for PT

Determining the prevalence of magnetic resonance imagingdiagnosed PT in middle-age community-based women and the fiagnosed PT in midd

Van der Worp et al. (2011a) Work-related etiological factor identification for PT and the connection amongst work limitations and PT

Van der Worp et al. (2012) Identification of risk factors of PT in volleyball and basketball players

\begin{tabular}{|c|c|c|}
\hline Study design & Study population & Level of participation \\
\hline $\begin{array}{l}\text { Survey-based prospective } \\
\text { cohort study }\end{array}$ & Male and female & Not applicable \\
\hline Cross-sectional study & Male & Elite athletes \\
\hline Systematic review & Male and female & Elite and recreational athletes \\
\hline Prospective cohort study & Female & General population \\
\hline Online survey: descriptive & Male and female & Elite and recreational athletes \\
\hline Cross-sectional study & Male and female & Elite and recreational athletes \\
\hline
\end{tabular}

PT, Patellar tendinopathy. 
TABLE 3: Intrinsic and extrinsic risk factors for patellar tendinopathy.

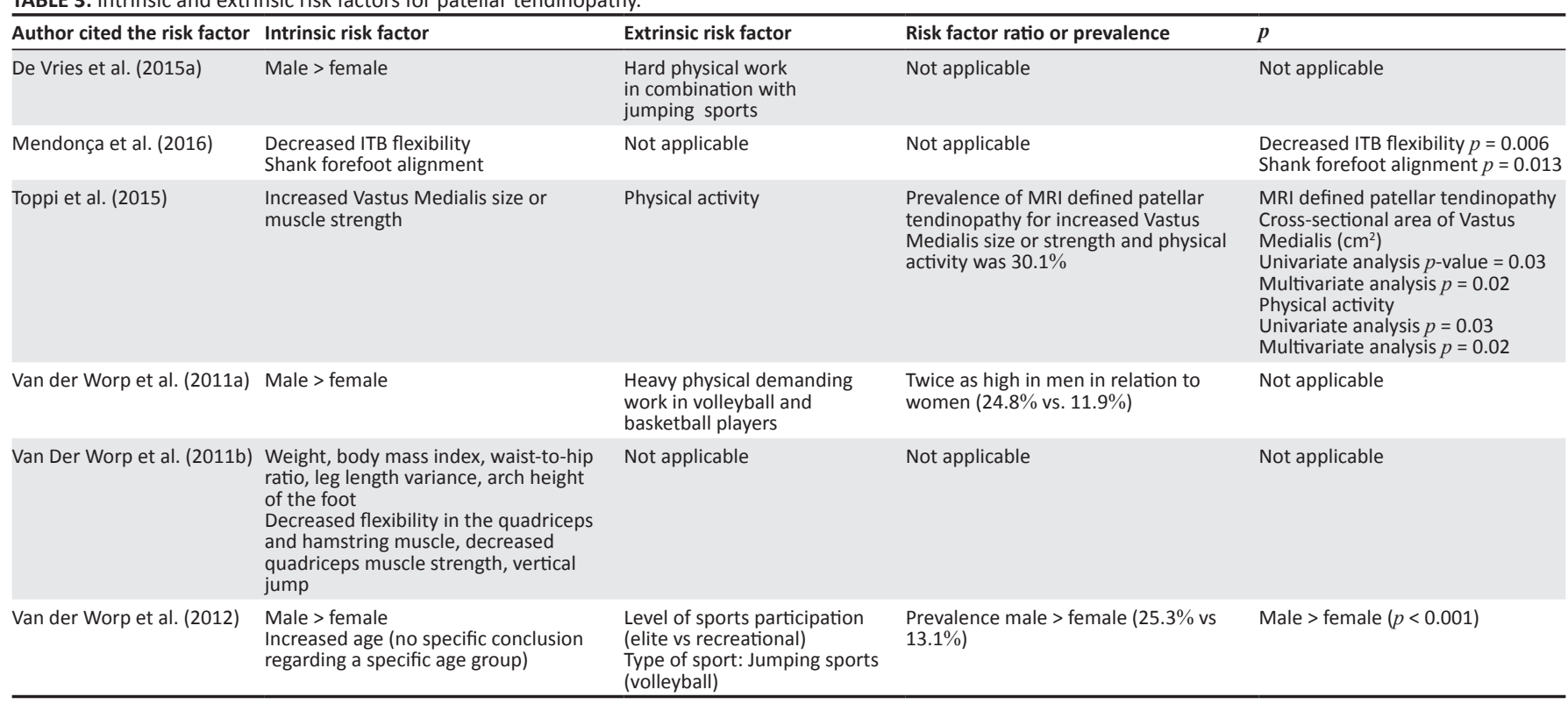

ITB, lliotibial band; MRI, magnetic resonance imaging.

of participation. A combination of elite $(66 \%)$ and recreational participants (50\%) was described in four articles, with one article having a general study participant population.

Eight intrinsic risk factors associated with PT were identified in the articles that described their intrinsic risk factors. Categorisation of these risk factors included three main intrinsic risk factors, namely gender (male > female), impaired lower limb muscle flexibility and muscle strength. The other identified intrinsic risk factors were body composition, leg length variances, anatomy of the foot, lower patellar pole and age of the study participants.

Five extrinsic risk factors for PT were identified with the main extrinsic risk factor being the common prevalence of PT in sports that involve jumping (50\%). The additional four extrinsic risk factors were heavy physical work in combination with jumping sports, level of sport participation (elite vs recreational level), physical activity and type of sport.

\section{Discussion}

PT is a well-recognised pathology with an inclusive aetiology of intrinsic non-modifiable and intrinsic and extrinsic modifiable risk factors that are directly linked to overloading of the patellar tendon (Hägglund et al. 2011). It is still unclear and debateable as to which particular mechanisms might be responsible for influencing intrinsic systemic factors for the risk of developing PT (Malliaras \& O'Neill 2017). According to the outcomes in this systematic review, gender (Morton et al. 2017) and age were non-modifiable intrinsic risk factors for PT. Gender was found to be a statistically similar finding to that reported by De Vries et al. (2015a), Van Der Worp et al. (2011b) and Van der Worp et al. (2012) that men are more likely to develop PT. This finding is also similar to results reported by Rudavsky and Cook (2014), indicating that male participants have a greater risk of sustaining PT than female participants. It is believed that oestrogen in female participants may act as a protective mechanism and this may explain the discrepancy between genders, although this needs further investigation (Torres, Zgonis \& Bernstein 2013).

The second non-modifiable intrinsic risk factor was increased age, granted that there was no conclusion regarding a specific age group in the results. The evidence shows that age may be associated with altered cellular activity in the patellar tendon, muscular function and mechanical properties (Malliaras \& $\mathrm{O}^{\prime}$ Neill 2017) and these age-related changes increase the predisposition of the general population to develop PT (Murtaugh \& Ihm 2013).

In general, the anatomy and biomechanics of each individual athlete or person have an influence on the risk of developing PT (Malliaras \& O'Neill 2017). Therefore, different morphologies of the foot (Reinking 2016), place increased strain and load on the patellar tendon (Schwartz, Watson \& Hutchinson 2015). In terms of leg length variance, the longer leg is generally associated with PT pathology with no other specific explanation (Van der Worp et al. 2011b) as to whether this is an anatomical or functional discrepancy. A lower patellar pole might be explained by the impingement theory, which implies the irritation of the patellar tendon on different knee flexion degrees (Torres et al. 2013). These three intrinsic risk factors for PT might be modified and improved in rehabilitation, but this has not been demonstrated in studies. One possible option in the rehabilitation of PT is the use of strapping or orthosis, which may potentially have a positive effect (De Vries et al. 2015b).

There are a further eight modifiable risk factors for PT which can be either intrinsic or extrinsic. Impaired quadriceps, hamstring and iliotibial band muscle flexibility is an intrinsic risk factor for PT (Malliaras \& O'Neill 2017; Reinking 2016; Schwartz et al. 2015). Muscle stiffness is 
related to general overuse of muscles, especially muscles in the lower limb and knee region (Brockmeyer et al. 2015) and injured athletes constantly present variations in flexibility deficits (O'Sullivan, McAuliffe \& Deburca 2012). As highlighted by Carvalho et al. (2012) and Rogan et al. (2013), lower limb stretching in athletes is necessary for optimal performance in sport, injury prevention and rehabilitation. Improving flexibility of the lower limbs has always been a focus area in the rehabilitation of PT (Rudvasky \& Cook 2015) and special attention is paid to improving the flexibility of the quadriceps femoris, hamstring and heel cord muscles by stretching programmes (Vetrano et al. 2013). This component of rehabilitation may benefit faster return to sport (O'Sullivan et al. 2012) by minimising symptoms (Rutland et al. 2010).

Impaired muscle strength of the quadriceps femoris increases the likelihood of developing PT (Rudvasky \& Cook 2015). The rationale behind this is that lower leg muscle strength, especially weakness surrounding the knee joint, contributes to patellar tendon strain by the abnormal distribution of load and malalignment of patellar tracking (Torres et al. 2013). To address this, it is advised that when strengthening is commenced during rehabilitation, one should start with isometric strengthening of the quadriceps muscle because of its pain inhibition (Rio et al. 2015). Once the pain is under control, progression to eccentric exercise can be started using the load tolerance principle (Malliaras et al. 2015). Eccentric exercise has been a cornerstone in PT rehabilitation since the 1980s (Steunebrink et al. 2013) and it is a popular (Saithna et al. 2012), extensively investigated therapeutic treatment for PT (Murtaugh \& Ihm 2013) that improves functionality (Sosa et al. 2014).

Body weight and body mass index are modifiable intrinsic risk factors for PT. The connection between body composition and the risk for PT is multifaceted and not simply related to loading of the tendon, thus outlining a complexity of confounding interconnected factors (Malliaras \& O'Neill 2017). Excessive body weight is responsible for an amplified patellar tendon load (Malliaras \& O'Neill 2017) and these combined factors affect microvascularity, which is directly linked to the development of PT (Murtaugh \& Ihm 2013).

Irrespective of the type of sport or activity that athletes or the general population participate in, musculoskeletal injuries are a reality (Saragiotto, Di Pierro \& Lopes 2013). Repetitive mechanical overload, an extrinsic risk factor (Rowan \& Drouin 2013), influences the patellar tendon in surpassing its reparative capacity (Rosso et al. 2015; Rutland et al. 2010), resulting in tendon failure and the development of PT (Malliaras \& O'Neill 2017). These risk factors for PT decrease the patellar tendon's ability to tolerate load over time, which is the typical nature of PT onset (Rosso et al. 2015; Rutland et al. 2010). This, again, highlights the direct connection between the development of PT and the load on the tendon which can be modified with a precise approach in rehabilitation (Vetrano et al. 2013).
Jumping sports participation is an important risk factor (De Vries et al. 2015a; Van der Worp et al. 2011a; Van der Worp et al. 2012), although the estimation of the actual prevalence of PT in specific sports that include jumping has not been identified. This is because of athletes continuing with sport participation even though they experience mild to moderate symptoms. Nonetheless, there is clear evidence that the prevalence of PT in jumping sports is alarmingly high because of the nature of this activity (Schwartz et al. 2015). Vertical jumping overloads the patellar tendon and seems to be because of the velocity and acceleration of ankle dorsiflexion and acceleration of knee flexion (Janssen, Steele \& Brown 2015).

The prevalence of PT fluctuates according to the specific jumping sport and between elite and recreational athletes (Rudavsky \& Cook 2014). A potential explanation for the prevalence of PT in elite athletes is that they are exposed to several loading and unloading time frames of the patellar tendon because of seasonal breaks and injuries over the years. This progressively decreases the capacity of the patellar tendon to tolerate load, and it becomes vulnerable with any overloading of the tendon, even with simple and minor adaptations in the training regimen (Rudavsky \& Cook 2014). On the other hand, one of the main reasons why recreational athletes sustain PT is rapid overloading of the patellar tendon. This overwhelms the tendon and also its capacity to recover, resulting from amplified physical activity, frequency or intensity and the use of incorrect equipment (Torres et al. 2013).

Unfortunately, PT is also prevalent in the general ageing population because of changes in the patellar tendon anatomical structure (Longo et al. 2011), and a quick run across the road or a day gardening may be adequate to overload the patellar tendon and symptoms will appear (Malliaras \& $\mathrm{O}^{\prime}$ Neill 2017). This agrees with the findings of age as a non-modifiable intrinsic risk factor for PT.

The secondary aim of the study was to initiate the development of a tool to screen for risk factors, as well as to potentially serve as an outcome measure in the rehabilitation of PT. This may contribute to the refining of PT management in terms of risk factor identification and determining and monitoring pain and function over time. A further possibility of the proposed screening tool in PT (Figure 2) is to potentially increase the awareness of further PT research in the healthcare community, particularly the development of validated PT screening tools.

The identified non-modifiable and modifiable intrinsic and extrinsic risk factors in this systematic review (despite the small sample size of the included articles) are all potentially relevant to the pathology and were used as the basis for the proposed PT screening tool (Figure 2). To broaden the tool to become an outcome measure (Scott et al. 2013), it is suggested that the proposed PT screening tool is combined with two subjective outcome measures and a pain provocation test for 
Patellar Tendinopathy Screening tool

\begin{tabular}{|l|}
\hline Name: \\
\hline Type of Sports Participation: \\
\hline
\end{tabular}

\section{Intrinsic risk factors}

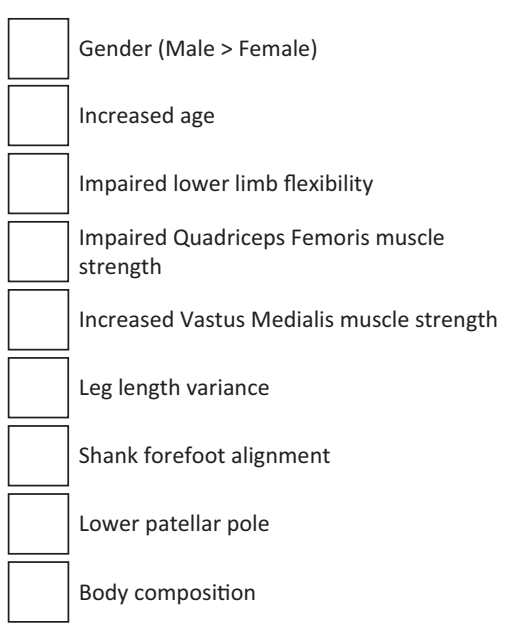

Outcome measures

VAS

VISA-P

Pain provocation test
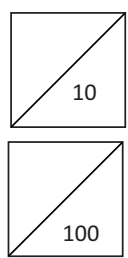

\section{Extrinsic risk factors}

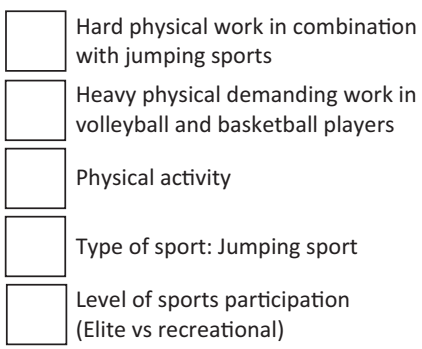

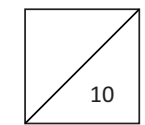

Comments:

FIGURE 2: Proposed Patellar Tendinopathy Screening tool.

load tolerance of the patellar tendon. According to Vetrano et al. (2013), the Victorian Institute of Sports Assessment for Patellar Tendinopathy questionnaire (VISA-P) and Visual Analog Scale (VAS) are well-known subjective questionnaires used extensively in PT rehabilitation. Independently, these outcome measurements have good validity and reliability for application in many clinical settings, including PT rehabilitation (De Vries et al. 2013; Gosens et al. 2012; 
Hernandez-Sanchez et al. 2017; Malliaras et al. 2015). The VAS is a sensitive, subjective one-dimensional questionnaire that estimates pain intensity in pathology (Hawker et al. 2011). It can measure the athlete's initial pain experience, pain during rehabilitation and offers precise indications of pain intensity and variations in pain (Vetrano et al. 2013). Patellar tendinopathy symptoms are difficult to quantify, and the VISA-P questionnaire is a disease-specific published clinical scale validated for PT, which contributes to its functional assessment (Vetrano et al. 2013).

Inclusion of a pain provocation test (Malliaras et al. 2015) may add considerable value to the evaluation of symptoms on a daily basis (Rudavsky \& Cook 2014), which is helpful in rehabilitation. Determining pain response in PT in terms of aggravation of symptoms at baseline, and during and after loading of energy-storage activities, is a major aspect in the rehabilitation and management of PT pathology in order to make load adaptations. This can be achieved by a $24-\mathrm{h}$ pain provocation test (Malliaras et al. 2015), which consists of a functional assessment test (single-leg decline squat test) (Rudavsky \& Cook 2014). If the pain on the provocation test returns to baseline in a $24-\mathrm{h}$ period, it implies that the patellar tendon pain is 'stable' and that the tendon has tolerated the load (Malliaras et al. 2015). This test can be advanced to a maximal vertical jump or triple-hop test during the returnto-sport phase of rehabilitation (De Vries et al. 2015b; Malliaras et al. 2015). According to Malliaras et al. (2015), the load tolerance principle is the single most important part of PT rehabilitation.

According to the authors' knowledge, there are no other PT screening tools. The proposed PT screening tool may possibly be useful in rehabilitation, as it includes a dual function of outlining likely intrinsic and extrinsic risk factors for the development of PT, the estimation of pain and functional impairments and is not indicated for any specific population. The value of the proposed PT screening tool will only be verified if properly tested in appropriate studies (Bishop 2008; McCreesh \& Lewis 2013).

\section{Strength, limitation and recommendations}

The strength of this systematic review is that the intrinsic and extrinsic risk factors for PT have been identified. Risk factor identification promotes the development and implementation of prevention strategies in the management of this condition. The evidence on the risk factors was used to suggest a proposed PT screening tool which will need to be tested in appropriate studies. A limitation of this systematic review was the limited number of included articles and a probable reason might be the explicit exclusion of youth populations. Another limitation was the lack of randomised clinical trials to validate the results.

\section{Conclusion}

Intrinsic and extrinsic risk factors for PT were identified in this systematic review. This evidence, as well as appropriate literature, formed the basis for the formulation of a proposed PT screening tool which will require testing to determine its usefulness.

\section{Acknowledgements Competing interests}

The authors declare that they have no financial or personal relations that may have inappropriately influenced them in writing the article.

\section{Authors' contributions}

S.M. and F.F.C. compiled the systematic review, discussed the construction of the article, screened the literature according to the eligibility criteria and analysed the data in order to formulate the checklist described in the article. S.M. wrote the article with the advice and editing of the co-author.

\section{References}

Abat, F. \& Sanchez-lbañez, J.M., 2014, 'Patellar tendinopathy: A critical review of current therapeutic options', Open Access Journal of Sports Medicine 2(1), 1-4.

Bishop, D., 2008, 'An applied research model for the sport sciences', Sports Medicine 38(3), 253-263. https://doi.org/10.2165/00007256-200838030-00005

Brockmeyer, M., Diehl, N., Schmitt, C., Kohn, D.M. \& Lorbach, O., 2015, 'Results of surgical treatment of chronic patellar tendinosis (jumper's knee): A systematic review of the literature', Journal of Arthroscopic \& Related Surgery 31(12), 24242429. https://doi.org/10.1016/j.arthro.2015.06.010

Brukner, P., Clarsen, B., Cook, J., Cools, A., Crossley, K., Hutchinson, M. et al., 2017, Bruckner and Kahn's clinical sports medicine, 5th edn., McGraw-Hill Education, Sydney, Australia.

Carvalho, F.L., Carvalho, M.C., Simão, R., Gomes, T.M., Costa, P.B., Neto, L.B. et al., 2012, 'Acute effects of a warm-up including active, passive, and dynamic stretching on vertical jump performance', Journal of Strength and Conditioning Research 26(9), 2447-2452. https://doi.org/10.1519/JSC.0b013e31823f2b36

Celebi, M.M., Köse, K., Akkava, Z. \& Zergeroglu, A.M., 2016, 'Cross-cultural adaptation of VISA-P score for patellar tendinopathy in Turkish population', Springerplus 5(1), 1453-1459. https://doi.org/10.1186/s40064-016-3100-x

De Vries, A.J., Van den Akker-Scheek, I., Diercks, R.L., Zwerver, J. \& Van der Worp, H., 2013, 'Effect of patellar strap and sports tape on jumper's knee symptoms: Protocol of a randomised controlled trial', Journal of Physiotherapy 59(4), 270285. https://doi.org/10.1016/S1836-9553(13)70204-2

De Vries, A.J., Van der Worp, H., Diercks, R.I., Van den Akker-Scheek, I. \& Zwerver, J., 2015a, 'Risk factors for patellar tendinopathy in volleyball and basketball players: A survey-based prospective cohort study', Scandinavian Journal of Medicine and Science in Sports 25(5), 678-684. https://doi.org/10.1111/sms.12294

De Vries, A., Zwerver, J., Diercks, R., Tak, I., Van Berkel, A., Van Cingel, R. et al., 2015b, 'Effect of patellar strap and sports tape on pain in patellar tendinopathy: $A$ randomised controlled trial' Scandinavian Journal of Medicine and Science in Sports 26(10), 1217-1224. https://doi.org/10.1111/sms.12556

De Vries, A.J., Koolhaas, W., Zwerver, J., Diercks, R.L., Nieuwenhuis, K., Van der Worp, $\mathrm{H}$. et al., 2017, 'The impact of patellar tendinopathy on sport and work performance in active athletes', Research in Sports Medicine 25(3), 253-265. https://doi.org/10.1080/15438627.2017.1314292

Gosens, T., Den Oudsten, B.L., Fievez, E., Van 't Spijker, P. \& Fievez, A., 2012, 'Pain and activity levels before and after platelet-rich plasma injection treatment of patellar tendinopathy: A prospective cohort study and the influence of previous treatments', International Orthopaedics 36(9), 1941-1946. https://doi.org/10.1007/s00264012-1540-7

Hawker, G.A., Mian, S., Kendzerska, T. \& French, M., 2011, 'Measures of adult pain Visual Analog Scale for Pain (VAS Pain), Numeric Rating Scale for Pain (NRS Pain), McGill Pain Questionnaire (MPQ), Short-Form McGill Pain Questionnaire (SFMPQ), Chronic Pain Grade Scale (CPGS), Short Form-36 Bodily Pain Scale (SF-36 BPS), and Measure of Intermittent and Constant Osteoarthritis Pain (ICOAP)', Arthritis Care and Research 63(Suppl 11), S240-S252.

Hägglund, M., Zwerver, J. \& Ekstrand, J., 2011, ‘Epidemiology of patellar tendinopathy in elite male soccer players' American Journal of Sports Medicine 39(9), 1906-1911. https://doi.org/10.1177/0363546511408877

Hernandez-Sanchez, S., Abat, F., Hidalgo, M.D., Cuesta-Vargas, A.I., Segarra, V. Sanchez-Ibañez, J.M. et al., A., 2017, 'Confirmatory factor analysis of VISA-P scale and measurement invariance across sexes in athletes with patellar tendinopathy', Journal of Sport and Health Science 6(3), 365-371. https://doi.org/10.1016/j. jshs.2016.01.020

Janssen, I., Steele, J.R. \& Brown, N.A.T., 2015, 'Landing technique is related to patellar tendon loading during a volleyball block jump movement', in F. Colloud, $M$. Domalain \& T. Monnet (eds.), Injuries/rehabilitation: 33rd International Conference Domalain \& T. Monnet (eds.), Injuries/rehabilitation: 33rd International Conference
on Biomechanics in Sports, Poitiers, France, June 29-July 3, 2015, viewed 11 June on Biomechanics in Sports, Poitiers, France, June 29-July 3, 2015, view
2018, from https://ojs.ub.uni-konstanz.de/cpa/article/view/6407/5774 
Liberati, A., Altman, D.G., Tetzla, J., Mulrow, C. \& Gøtzsche, P.C., 2009, 'The PRISMA statement for reporting systematic reviews and meta-analyses of studies that evaluate health care interventions: Explanation and elaboration', PLOS Medicine 6(7), e1000100. https://doi.org/10.1371/journal.pmed.1000100

Longo, U.G., Rittweger, J., Garau, G., Radonic, B., Gutwasser, C., Gilliver, S.F. et al., 2011, 'Patellar tendinopathy in master track and field athletes: Influence of impact profile, weight, height, age and gender' Knee Surgery, Sports Traumatology, Arthroscopy 19(3), 508-512. https://doi.org/10.1007/s00167-010-1314-y

Malliaras, P., Cook, J., Purdam, C. \& Rio, E., 2015, 'Patellar tendinopathy: Clinical diagnosis, load management, and advice of challenging case presentations Journal of Orthopaedic and Sports Physical Therapy 45(11), 887-898. https://doi. org/10.2519/jospt.2015.5987

Malliaras, P. \& O'Neill, S., 2017, 'Potential risk factors leading to tendinopathy', Apunts Medicina de L'esport 52(194), 71-77. https://doi.org/10.1016/j. apunts.2017.06.001

McCreesh, K. \& Lewis, J., 2013, 'Continuum model of tendon pathology: Where are we now?', International Journal of Experimental Pathology 94(4), 242-247. https:// doi.org/10.1111/iep.12029

Mendonça, L.D., Verhagen, E., Bittencourt, N.F, Gonçalves, G.G., Ocarino, J.M. \& Fonseca, S.T., 2016, 'Factors associated with the presence of patellar tendon abnormalities in male athletes', Journal of Science and Medicine in Sport 19(5), 389-394. https://doi.org/10.1016/j.jsams.2015.05.011

Moher, D., Liberati, A., Tetzlaff, J., Altmans, D.G. \& PRISMA Group, 2009, 'Preferred reporting items for systematic reviews and meta-analyses: The PRISMA statement', Public Library of Science Medicine 6(7), e1000097.

Morgan, S., Janse van Vuuren, E.C. \& Coetzee, F.F., 2016, 'Causative factors and rehabilitation of patellar tendinopathy: A systematic review', South African Journal of Physiotherapy 72(1), 1-11. https://doi.org/10.4102/sajp.v72i1.338

Morton, S., Williams, S., Valle, X., Diaz-cueli, D., Malliaras, P. \& Morrissey, D., 2017 'Patellar tendinopathy and potential risk factors: An international database of cases and controls', Clinical Journal of Sport Medicine 27(5), 468-474. https://doi. org/10.1097/JSM.0000000000000397

Murtaugh, B. \& Ihm, J.M., 2013, 'Eccentric training for the treatment of tendinopathies', Current Sports Medicine Reports 12(3), 175-182. https://doi.org/10.1249/ JSR.0b013e3182933761

National Institute for Health and Care Excellence (NICE), 2012, Methods for the development of NICE public health guidance. Appendix F Quality appraisal checklist - Quantitative intervention studies, viewed 10 May 2018, from https:// www.nice.org.uk/process/pmg4/chapter/appendix-f-quality-appraisal-checklistquantitative-intervention-studies.

O'Sullivan, K., McAuliffe, S. \& Deburca, N., 2012, 'The effects of eccentric training on lower limb flexibility: A systematic review, British Journal of Sports Medicine 46(12), 838-845. https://doi.org/10.1136/bjsports-2011-090835

Peters, J.A., Zwerver, J., Diercks, R.L., Elferink-Gemser, M.T. \& Van den Akker-Scheek, I., 2016, 'Preventive interventions for tendinopathy: A systematic review', Journal of Science and Medicine in Sport 19(3), 205-211. https://doi.org/10.1016/j. jsams.2015.03.008

Reinking, M.F., 2016, 'Current concepts in the treatment of patellar tendinopathy', International Journal of Sports Physical Therapy 11(6), 854-866.

Rio, E., Kidgell, D., Purdam, C., Gaida, J., Moseley, G.L., Pearce, A.J. et al., 2015, 'Isometric exercise induces analgesia and reduces inhibition in patellar tendinopathy', British Journal of Sports Medicine 49(19), 1277-1283. https://doi. tendinopathy', British Journal of Sports
org/10.1136/bjsports-2014-094386

Rodriguez-Merchan, E.C., 2013, 'The treatment of patellar tendinopathy', Journal of Orthopaedics and Traumatology 14(2), 77-81. https://doi.org/10.1007/s10195012-0220-0

Rogan, S., Wüst, D., Schwitter, T. \& Schmidtbleicher, D., 2013, 'Static stretching of the hamstring muscle for injury prevention in football codes: A systematic review', Asian Journal of Sports Medicine 4(1), 1-9.

Rosso, F., Bonasia, D.E., Cottino, U., Dettoni, F., Bruzzone, M. \& Rossi, R., 2015, 'Patellar tendon: From tendinopathy to rupture', Asian-Pacific Journal of Sports Medicine, Arthroscopy, Rehabilitation and Technology 2(4), 99-107. https://doi.org/10.1016/j. asmart.2015.07.001

Rowan, T.L. \& Drouin, J.L., 2013, 'A multidisciplinary approach including the use of platelet-rich plasma to treat an elite athlete with patellar tendinopathy - A case report', Journal of the Canadian Chiropractic Association 57(4), 301-309.
Rudavsky, A. \& Cook, J., 2014, 'Physiotherapy management of patellar tendinopathy (jumper's knee)', Journal of Physiotherapy 60(3), 122-129. https://doi.org/10.1016/j. jphys.2014.06.022

Rutland, M., O'Connell, D., Brismée, J.M., Sizer, P., Apte, G. \& O'Connell, J., 2010, 'Evidence-supported rehabilitation of patellar tendinopathy', North American Journal of Sports Physical Therapy 5(3), 166-178.

Saithna, A., Gogna, R., Baraza, N., Modi, C. \& Spencer, S., 2012, 'Eccentric exercise protocols for patella tendinopathy: Should we really withdraw athletes from sport? A systematic review', Open Orthopaedics Journal 6(3), 553-557 https:// doi.org/10.2174/1874325001206010553

Saragiotto, B.T., Di Pierro, C. \& Lopes, A.D., 2013, 'Risk factors and injury prevention in elite athletes: A descriptive study of the opinions of physical therapists, doctors and trainers', Brazilian Journal of Physical Therapy 8(2), 137-143. https://doi. org/10.1590/S1413-35552012005000147

Schwartz, A., Watson, J.N. \& Hutchinson, M.R., 2015, 'Patellar tendinopathy', Sports Health 7(5), 415-420. https://doi.org/10.1177/1941738114568775

Scott, A., Docking, S., Vicenzino, B., Alfredson, H., Zwerver, J., Lundgreen, K. et al. 2013, 'Sports and exercise-related tendinopathies: A review of selected topical issues by participants of the second International Scientific Tendinopathy Symposium (ISTS) Vancouver 2012', British Journal of Sports Medicine 47(9), 536-544. https://doi.org/10.1136/bjsports-2013-092329

Shea, B.J., Hamel, C., Wells, G.A., Bouter, L.M., Kristjansson, E., Grimshaw, J. et al., 2009, 'AMSTAR is a reliable and valid measurement tool to assess the methodological quality of systematic reviews', Journal of Clinical Epidemiology 62(10), 1013-1020. https://doi.org/10.1016/j.jclinepi.2008.10.009

Sosa, C., Lorenzo, A., Jiménez, S.L. \& Bonfanti, N., 2014, 'Eccentric exercise in treatment of patellar tendinopathy in high level basketball players. A randomized clinical trial', Journal of Strength and Conditioning Research 28(11), 1.

Steunebrink, M., Zwerver, J., Brandsema, R., Groenenboom, P., Van den Akker-Scheek, I. \& Weir, A., 2013, 'Topical glyceryl trinitrate treatment of chronic patellar tendinopathy: A randomised, double-blind, placebo-controlled clinical trial', British Journal of Sports Medicine 47(1), 34-39. https://doi.org/10.1136/bjsports-2012-091115

Stuhlman, C.R., Stowers, K., Stowers, L. \& Smith, J., 2016, 'Current concepts and the role of surgery in the treatment of jumper's knee', Orthopaedics 39(6), e1028e1035. https://doi.org/10.3928/01477447-20160714-06

Torres, S., Zgonis, M.H. \& Bernstein, J., 2013, 'Patellar tendinopathy', Hospital Physician Board Review Manual 8(4), 6-19.

Toppi, J., Fairley, J., Cicuttini, F.M., Cook, J., Davis, S.R., Bell, R.J. et al., 2015, 'Factors associated with magnetic resonance imaging defined patellar tendinopathy in community-based middle-aged women: A prospective cohort study', BMC Musculoskeletal Disorders 16, 184-190. https://doi.org/10.1186/s12891-015-0645-8

Van Ark, M., Cook, J.L., Docking, S.I., Zwerver, J., Gaida, J.E., Van den Akker-Scheek, I. et al., 2016, 'Do isometric and isotonic exercise programs reduce pain in athletes with patellar tendinopathy in-season? A randomised clinical trial', Journal of Science and Medicine in Sport 19(9), 702-706. https://doi.org/10.1016/j.jsams.2015.11.006

Van der Worp, H., Van Ark, M., Roerink, S., Pepping, G.J., Van den Akker-Scheek, I. \& Zwerver, J., 2011b, 'Risk factors for patellar tendinopathy: A systematic review of the literature', British Journal of Sports Medicine 45(5), 446-452. https://doi. org $/ 10.1136 /$ bjsm.2011.084079

Van der Worp, H., Van Ark, M., Zwerver, J. \& Van den Akker-Scheek, I., 2012, 'Risk factors for patellar tendinopathy in basketball and volleyball players: A crosssectional study'. Scandinavian Journal of Medicine and Science in Sport 22(6) 783-790. https://doi.org/10.1111/j.1600-0838.2011.01308.x

Van der Worp, H., Zwerver, J., Kuijer, P.P.F.M., Frings-Dresen, M.H.W. \& Van den AkkerScheek, I., 2011a, 'The impact of physically demanding work of basketball and volleyball players on the risk for patellar tendinopathy and on work limitations' Journal of Back and Musculoskeletal Rehabilitation 24(1), 49-55. https://doi. org/10.3233/BMR-2011-0274

Vetrano, M., Castorina, A., Vulpiani, M.C., Baldini, R., Pavan, A. \& Ferretti, A., 2013 'Platelet-rich plasma versus focused shock waves in the treatment of jumper's knee in athletes' American Journal of Sports Medicine 41(4), 795-803. https://doi. org/10.1177/0363546513475345

Winn, J., 2013, A systematic review to assess the methodological quality of intervention research designed to increase physical activity in children with autism spectrum disorders, viewed 11 June 2018, from http://stars.library.ucf. edu/honorstheses1990-2015/1485 


\section{Appendix 1:}

\section{Exclusion of articles because of unsuitability based on title screening $(n=49)$}

Abat, F., Diesel, W.J., Gelber, P.E., Polidori, F., Monllau, J.C. \& Sanchez-ibanez, J.M., 2014, 'Effectiveness of the intratissue percutaneous electrolysis (EPI) technique and isoinertial eccentric exercise in the treatment of patellar tendinopathy at two years follow-up', Muscles, Ligaments and Tendons Journal 4(2), 188-193.

Abstracts of the International Federation of Associations of Anatomists, 2014, viewed 12 September 2016, from http:// www.csas.org.cn/ifaa2014/AANAT.pdf

Benner, R.W., Shelbourne, K.D. \& Gray, T., 2016, 'The degree of knee extension does not affect postoperative stability or subsequent graft tear rate after anterio cruciate ligament reconstruction with patellar tendon autograft', American Journal of Sports Medicine 44 (4), 844-849.

Biernat, R., Trzaskoma, Z., Trzaskoma, L. \& Czaprowski, D., 2014, 'Rehabilitation protocol for patellar tendinopathy applied among 16- to 19-year old volleybal players', Journal of Strength and Conditioning Research 28(1), 43-52.

Brockmeyer, M., Diehl, N., Schmitt, C., Kohn, D.M. \& Lorbach, O., 2015, 'Results of surgical treatment of chronic patellar tendinosis (jumper's knee): A systematic review of the literature', The Journal of Arthroscopic \& Related Surgery 31(12), 2424-2429.

Camargo, P.R., Alburquerque-Sendin, F. \& Salvini, T.F., 2014, 'Eccentric training as a new approach for rotator cuff tendinopathy: Review and perspectives', World new approach for rotator cuff tend
Journal of Orthopedics 5(5), 634-644

Campbell, R.S.D. \& Dunn, A.J., 2012, 'Radiological interventions for soft tissue injuries in sport', British Journal of Radiology 85(1016), 1186-1193.

Clark, N.T.M., Bourque, R.D., Schilling, J. \& Mckeon, P., 2014, 'Treatment of patellofemoral pain syndrome in a track athlete', International Journal of Athletic Therapy \& Training 19(1), 27-31.

Ellenbecker, T.S., Pieczynski, T.E. \& Davies, G.J., 2010, 'Rehabilitation of the elbow following sports injury', Clinics in Sports Medicine 29(1), 33.

Filardo, G., Kon, E., Di Matteo, B., Di Martino, A., Tesei, G., Pelotti, P. et al., 2014 'Platelet-rich plasma injections for the treatment of refractory Achilles tendinopathy: Results at 4 years', Blood Transfusion 12(4), 533-540.

Filardo, G., Kon, E., Di Matteo, B., Pelotti, P., Di Martino, A. \& Marcacci, M., 2013 'Platelet-rich plasma for the treatment of patellar tendinopathy: Clinical and imaging findings at medium-term follow-up', International Orthopaedics 37(8), 1583-1589.

Gill, T.J., Carroll, K.M. \& Hariri, S., 2013, 'Open patellar tendon debridement for treatment of recalcitrant patellar tendinopathy: Indications, technique, and clinical outcomes after a 2-year minimum follow-up' Sports Health: A Multidisciplinary Approach 5(3), 276

Hadzic, V., Sattler, T., Markovic, G., Veselko, M. \& Dervisevic, E., 2010, 'The isokinetic strength profile of quadriceps and hamstrings in elite volleyball players', Isokinetics and Exercise Science 18(1), 31-37.

Hall, R., Barber Foss, K., Hewett, T.E. \& Myer, G.D., 2015, 'Sport specialization's association with an increased risk of developing anterior knee pain in adolescent female athletes', Journal of Sport Rehabilitation 24(1), 31-35.

Hall, M.M. \& Rajasekaran, S., 2016, 'Ultrasound-guided scraping for chronic patellar tendinopathy: A case presentation', Journal of Injury, Function, and Rehabilitation $8(6), 593-596$.

Hernandez, V.P., Varela, S.M. \& Moraleda, B.R., 2011, 'Proposal for functional recovery from ruptured anterior cruciate ligament in soccer' Revista Internacional de Medicina y Ciencias de la Actividad Fisica y del Deporte 11(43), 573-591.

Goom, T.S., Malliaras, P., Reiman, M.P. \& Purdam, C.R., 2016, 'Proximal hamstring tendinopathy: Clinical aspects of assessment and management', Journal of Orthopaedic \& Sports Physical Therapy 46(6), 483.

Kaux, J.F., Bruyere, O., Croisier, J.L., Forthomme, B., Le Goff, C. \& Crielaard, J.M., 2015 'One-year follow-up of platelet-rich plasma infiltration to treat chronic proximal patellar tendinopathies', Acta Orthopaedica Belgica 81(2) 251-256.

Kaux, J.F., Croisier, J.L., Bruyère, O., Rodriguez, C., Daniel, C. \& Godon, B., 2013 , 'Platelet-rich plasma (PRP) to treat chronic upper patellar tendinopathies', British Journal of Sports Medicine 47(10), 6.

Kaux, J.F., Croisier, J.L., Bruyère, O., Rodriguez De La Cruz, C., Forthomme, B., Brabant G. et al., 2015, 'One injection of platelet-rich plasma associated to a submaximal eccentric protocol to treat chronic jumper's knee', The Journal of Sports Medicine and Physical Fitness 55(9), 953-961.

Kaux, J.F., Croisier, J.L., Forthomme, B., Le Goff, C., Delcour, S., Gothot, A. \& Crielaard, J.M., 2015, 'Exploring the effect of a second closely-timed infiltration of platelet-rich plasma to treat jumper's knees', Annals of Physical \& Rehabilitation Medicine 58, 68

Kaux, J.-F., Forthomme, B., Namurois, M.-H., Bauvir, P., Defawe, N., Delvaux, F. et al 2014 , 'Description of a standardized rehabilitation program based on sub-maximal eccentric following a platelet-rich plasma infiltration for jumper's knee', Muscles, Ligaments \& Tendons Journal 4(1), 85-89.

Kim, K.H. \& Kim, T.G., 2011, 'Effects of patellar tendinopathy on the marche-fente movement of female fencing fleuret players', Korean Journal of Sport Science 22(2), 1875-1883.

Kulig, K., Noceti-Dewit, L.M., Reischl, S.F. \& Landel, R.F., 2015, 'Physical therapists' role in prevention and management of patellar tendinopathy injuries in youth collegiate, and middle-aged indoor volleyball athletes', Brazilian Journal of Physical Therapy 19(5), 410-420.
Liddle, A.D. \& Rodríguez-Merchán, E.C., 2015, 'Platelet-rich plasma in the treatment of patellar tendinopathy', American Journal of Sports Medicine 43(10), 2583-2590.

Maffulli, N., Giai Via, A. \& Oliva, F., 2017, 'Revision surgery for failed patellar tendinopathy exploration' Sports Medicine and Arthroscopy Review 25(1), 36-40.

Maier, D., Bornebusch, L., Salzmann, G.M., Sudkamp, N.P. \& Ogon, P., 2013, 'Mid- and long-term efficacy of the arthroscopic patellar release for treatment of patellar tendinopathy unresponsive to nonoperative management', Arthroscopy-The Journal of Arthroscopic and Related Surgery 28(8), 1338-1345.

Marcheggiani, M., Giulio, M., Zaffagnini, S., Tsapralis, K., Alessandrini, E., Bonanzinga T. et al., 2013, 'Open versus arthroscopic surgical treatment of chronic proximal patellar tendinopathy. A systematic review', Knee Surgery, Sports Traumatology, Arthroscopy 21(2), 351-357

Martínez-Rodríguez, A., Moreno-Pérez, V., Roche, E. \& Vicente-Salar, N., 2013, 'Planificación dietética y rehabilitación a Largo Plazo de Jugadores profesionales de tenis y fútbol mediante una
of Human Movement $31,77$.

Morton, S., Otto, C., King, J., Perry, D., Crisp, T., Maffulli, N. et al., 2014, 'High volume image-guided injections for patellar tendinopathy: A combined retrospective and prospective case series', Muscles, Ligaments \& Tendons Journal 4(2), 214-219.

Muneta, T., Koga, H., JU, Y.J., Mochizuki, T. \& Sekiya, I., 2012, 'Hyaluronan injection therapy for athletic patients with patellar tendinopathy', Journal of Orthopaedic Science 17(4), 425-431.

Nanos, K.N. \& Malanga, G.A., 2015, 'Treatment of patellar tendinopathy refractory to surgical management using percutaneous ultrasonic tenotomy and platelet-rich plasma injection: A case presentation', Journal of Injury, Function, And Rehabilitation 7(12), 1300-1305.

Ogon, P., Izadpanah, K., Eberbach, H., Lang, G., Südkamp, N.P. \& Maier, D., 2017, 'Prognostic value of MRI in arthroscopic treatment of chronic patellar tendinopathy: A prospective cohort study', BioMed Central Musculoskeletal Disorders 18, 1-9.

Park, B.H., Seo, J.H., Ko, M.H. \& Park, S.H., 2013, 'Reliability and validity of the Korean version VISA-P Questionnaire for patellar tendinopathy in adolescent elite version VISA-P Questionnaire for patellar tendinopathy in adolesce
volleyball athletes', Annals of Rehabilitation Medicine 37(5), 698-705.

Pascarella, A., Alam, M., Pascarella, F., Latte, C., Di Salvatore, M.G. \& Maffulli, N., 2011, 'Arthroscopic management of chronic patellar tendinopathy', American Journal of Sports Medicine 39(9), 1975-1983.

Physical Therapy: News from the Foundation for Physical Therapy 2010, viewed 12 September 2016, from http:// ptjournal.apta.org/content/90/11/1697.full

Plinsinga, M.L., Brink, M.S., Vicenzino, B. \& Van Wilgen, C.P., 2015, 'Evidence of nervous system sensitization in commonly presenting and.persistent painful tendinopathies: A systematic review', Journal of Orthopaedic \& Sports Physical Therapy 45(11), 864-875.

Research Report Abstracts, Physiotherapy 2011, viewed 12 September 2016, from http://www.physiotherapyjournal.com/issue/S0031-9406(11)X0004-4

Rio, E., Kidgell, D., Moseley, G.L. \& Cook, J., 2016, 'Elevated corticospinal excitability in patellar tendinopathy compared with other anterior knee pain or no pain', Scandinavian Journal of Medicine \& Science in Sports 26(9), 1072-1079.

Ryan, M., Wong, A., Rabago, D., Lee, K. \& Taunton, J., 2011, 'Ultrasound-guided injections of hyperosmolar dextrose for overuse patellar tendinopathy: A pilot study', British Journal of Sports Medicine 45(12), 972-977.

Smith, J. \& Sellon, J.L., 2014, 'Comparing PRP injections with ESWT for athletes with chronic patellar tendinopathy', Clinical Journal of Sport Medicine: Official Journal of the Canadian Academy of Sport Medicine 24(1), 88-89.

Stuhlman, C.R., Stowers, K., Stowers, L. \& Smith, J., 2016, 'Current concepts and the role of surgery in the treatment of jumper's knee' (English), Orthopedics 39(6), e1028-e1035.

Sunding, K., Willberg, L., Werner, S., Alfredson, H., Forssblad, M. \& Fahlström, M., 2015, 'Sclerosing injections and ultrasound-guided arthroscopic shaving for patellar tendinopathy: Good clinical results and decreased tendon thickness after surgery-a medium-term follow-up study', Knee Surgery, Sports Traumatology, surgery-a medium-term follow-up
Arthroscopy 23(8), 2259-2268.

Van Ark, M., Van Den Akker-Scheek, I., Meijer, L.T.B. \& Zwerver, J., 2013, 'An exercisebased physical therapy program for patients with patellar tendinopathy after platelet-rich plasma injection' Physical Therapy in Sport 14(2), 124-130.

Van Duren, B., Pandit, H., Murray, D. \& Gill, H., 2015, 'Approximation of the functional kinematics of posterior stabilised total knee replacements using a two-dimensional sagittal plane patello-femoral model: Comparing model approximation to in vivo measurement', Computer Methods in Biomechanics \& Biomedical Engineering 18(11), 1191-1199.

Verrall, G., Schofield, S. \& Brustad, T., 2011, 'Chronic Achilles tendinopathy treated with eccentric stretching program', Foot \& Ankle International 32(9), 843-849.

Willberg, L., Sunding, K., Forssblad, M., Fahlström, M. \& Alfredson, H., 2011, 'Sclerosing polidocanol injections or arthroscopic shaving to treat patellar
tendinopathy/jumper's knee? A randomised controlled study', British Journal of tendinopathy/jumper's knee? A
Sports Medicine 45(5), 411-415.

Xu, H., Bloswick, D. \& Merryweather, A., 2015, 'An improved OpenSim gait model with multiple degrees of freedom knee joint and knee ligaments', Computer Methods in Biomechanics \& Biomedical Engineering 18(11), 1217-1224.

\section{Exclusion of articles because of unsuitability based on abstract screening $(n=21)$}

Bond, R.P. \& Snyckers, C.H., 2010. 'Management of sports overuse injuries of the lower limb: An evidence-based review of the literature', South African Orthopaedic Journal 9(2), 48-58. 
Caquot, J., Perrochon, A., Bugeaud, J.L., Dumélié, X., Duzou, F. \& Daviet, J.C., 2016, 'Cognitive representations on patellar tendinopathy at the basketball players of 'Cognitive representations on patellar tendinopathy at the basketball players of
the French training centers', Annals of Physical \& Rehabilitation Medicine 59, e16.

Caquot, J., Perrochon, A., Bugeaud, J.L., Dumélié, X., Pajon, M. \& Daviet, J.C., 2016 'Prevalence of pain below patella in the basketball players of the championship of France hope pro A', Annals of Physical \& Rehabilitation Medicine 59, e15-e16.

de Carlo, M. \& Armstrong, B., 2010, 'Rehabilitation of the knee following sports injury', Clinics in Sports Medicine 29(1), 81-106.

Dimnjaković, D., Bojanić, I., Smoljanović, T., Mahnik, A. \& Barbarić-Peraić, N., 2012 'Eccentric exercises in the treatment of overuse injuries of the musculoskeletal system', Lijecničkĭ Vjesnik 134(1-2), 29-41.

Eerkes, K., 2012, 'Volleyball Injuries', Current Sports Medicine Reports 11(5), 251-256.

Giombini, A., Dragoni, S., Di Cesare, A., Di Cesare, M., Del Buono, A. \& Maffulli, N., 2013, 'Asymptomatic achilles, patellar, and quadriceps tendinopathy: A 2013, 'Asymptomatic achilles, patellar, and quadriceps tendinopathy: A
longitudinal clinical and ultrasonographic study in elite fencers', Scandinavian Journal of Medicine \& Science in Sports 23(3), 311-316.

Gómez-Valero, S., García-Pérez, F., Flórez-García, M.T. \& Miangolarra-Page, J.C., 2017 'A systematic review of self-administered questionnaires for the functional assessment of patients with knee disabilities adapted into Spanish', Revista Espanola De Cirugia Ortopedica Y Traumatologia 61(2), 96-103.

Gordon, A.I., Distefano, L.J., Denegar, C.R., Ragle, R.B., Norman, J.R. \& Cheatham, S., 2014, 'College and professional women's basketball players' lower extremity injuries: A survey of career incidence', International Journal of Athletic Therapy \& Training 19(5), 25-33.

Hernandez-Sanchez, S., Hidalgo, M.D. \& Gomez, A., 2011, 'Cross-cultural adaptation of VISA-P Score for patellar tendinopathy in Spanish population', Journal of Orthopaedic \& Sports Physical Therapy 41(8), 581-591.

Kristensen, J. \& Franklyn-Miller, A., 2012, 'Resistance training in musculoskeletal rehabilitation: A systematic review', British Journal of Sports Medicine 46(10), 719-726.

Moura, D.L., Marques, J.P., Lucas, F.M. \& Fonseca, F.P., 2016, 'Simultaneous bilateral patellar tendon rupture', Revista Brasileira De Ortopedia 52(1), 111-114.

Pas, H.I.M.F.L., Moen, M.H., Haisma, H.J. \& Winters, M., 2017, 'No evidence for the use of stem cell therapy for tendon disorders: A systematic review', British Journal of Sports Medicine 51(13), 996-1002.

Plinsinga, M., Vuvan, V., Stephenson, A., Mellor, R., Heales, L., Van Wilgen, P. et al., 2017, 'Pain and psychological characteristics in patellar tendinopathy', Journal of Science \& Medicine in Sport 20, e20.

Rio, E., Kidgell, D., Moseley, G.L. \& Cook, J., 2015, 'Elevated corticospinal excitability in patellar tendinopathy compared with other anterior knee pain or no pain', Scandinavian Journal of Medicine \& Science in Sports 26(9), 1072-1079.

Rio, E., Van Ark, M., Docking, S., Moseley, G.L., Kidgell, D., Gaida, J.E. et al., 2017 Isometric contractions are more analgesic than isotonic contractions for patella tendon pain: An in-season randomized clinical trial',. Clinical Journal of Sport Medicine 27(3), 253-259.

Schwartz, A., Watson, J.N. \& Hutchinson, M.R., 2015, 'Patellar Tendinopathy', Sports Health 7(5), 415-420.

Wearing, S.C., Locke, S., Smeathers, J.E. \& Hooper, S.L., 2015, Tendinopathy alters cumulative transverse strain in the patellar tendon after exercise', Medicine \& Science in Sports \& Exercise 47(2), 264-271.

White, T. \& Clapis, P., 2010, 'Patellar tendonitis (jumper's knee) rehabilitation exercises' Computerized Registration System - Sports Medicine Advisor 1 viewed 20 August 2018, from, http://sportmed.com/wp-content/uploads/Combined 20 August 2018, from
Patellar_Tendinitis.pdf

Young, M.A., Cook, J.L., Purdam, C.R., Kiss, Z.S. \& Alfredson, H., 2010, 'Patellar tendon injury (jumper's knee) rehabilitation exercises: References', Computerized Registration System - Sports Medicine Advisor 1, viewed 20 August 2018, from sportmed.com/wp-content/uploads/Combined_Patellar_Tendinitis.pdf.

Zhang, Z.J., Ng, G.Y.F., Lee, W.C. \& Fu, S.N., 2017, 'Increase in passive muscle tension of the quadriceps muscle heads in jumping athletes with patellar tendinopathy', Scandinavian Journal of Medicine \& Science in Sports 10, 1099-1104.

\section{Exclusion of articles because of unsuitability based on full-text screening $(n=40)$}

Couppé, C., Kongsgaard, M., Aagaard, P., Vinther, A., Boesen, M., Kjær, M. et al., 2013 , 'Differences in tendon properties in elite badminton players with or without patellar tendinopathy', Scandinavian Journal of Medicine \& Science in Sports 23(2), 89-95.

Culvenor, A.G., Cook, J.L., Warden, S.J. \& Crossley, K.M., 2011, 'Infrapatellar fat pad size, but not patellar alignment, is associated with patellar tendinopathy', Scandinavian Journal of Medicine \& Science in Sports 21(6), 405-411.

Da Cunha, R.A., Dias, A.N., Santos, M.B. \& Lopes, A.D., 2012, 'Comparative study of two protocols of eccentric exercise on knee pain and function in athletes with patellar tendinopathy: Randomised controlled study', Revista Brasileira de Medicina do Esporte 18(3), 167-170.

De Vries, A., Zwerver, J., Diercks, R., Tak, I., Van Berkel, A., Van Cingel, R. et al., 2015b 'Effect of patellar strap and sports tape on pain in patellar tendinopathy: A randomised controlled trial', Scandinavian Journal of Medicine and Science in Sports 26(10), 1217-1224.

Dimitrios, S., 2015, 'There is lack of evidence to support the effectiveness of therapeutic ultrasound in the management of patellar tendinopathy', Physical Therapy Reviews 20(4), 268-269.
Dimitrios, S., Pantelis, M. \& Kalliopi, S., 2012, 'Comparing the effects of eccentric training with eccentric training and static stretching exercises in the treatment of patellar tendinopathy. A controlled clinical trial' Clinical Rehabilitation 26(5), 423-430.

Edouard, P., Cugy, E., Dolin, R., Morel, N. \& Steffen, K., 2016, 'An injury prevention program is able to reduce the number of injury complaints at medium-term in program is able to reduce the number of injury complaints at medic
athletics', Annals of Physical \& Rehabilitation Medicine 59, e17-e18.

Frizziero, A., Trainito, S., Oliva, F., Aldini, N.N., Masiero, S. \& Maffulli, N., 2014, 'The role of eccentric exercise in sport rehabilitation', British Medical Bulletin 110(1), $47-75$.

Goldman, R.B. \& Lentz, T.A., 2010, 'The use of eccentric overloading exercise for the treatment of patellar tendinosis in an Olympic-style weightlifter: A case report', Orthopaedic Practice 22(2:10), 76-82.

Ho, K.Y. \& Kulig, K., 2016, 'Changes in water content in response to an acute bout of eccentric loading in a patellar tendon with a history of tendinopathy: A case report', Physiotherapy Theory \& Practice 32(7), 566-570.

Jain, N., Kemp, S., Hayward, P. \& Murray, D.J., 2012, 'Effect of pre-season screening for patella tendinopathy: The findings of a professional football club', Muscles, Ligaments \& Tendons Journal 10

James, L., Kelly, V. \& Beckman, E., 2014, 'Injury risk management plan for volleybal athletes', Sports Medicine 44(9), 1185-1195.

Kulig, K., Noceti-Dewit, L.M., Reischl, S.F. \& Landel, R.F., 2015, 'Physical therapists' role in prevention and management of patellar tendinopathy injuries in youth, collegiate, and middle-aged indoor volleyball athletes', Brazilian Journal of Physical Therapy 19(5), 410-420.

Leal, C., Ramon, S., Furia, J., Fernandez, A., Romero, L. \& Hernandez-Sierra, L., 2015 'Current concepts of shockwave therapy in chronic patellar tendinopathy', International Journal of Surgery 24(B), 160-164.

Lee, W., Zhang, Z., Masci, L., Ng, G., Fu, S., Lee, W.C. et al.,2017, 'Alterations in mechanical properties of the patellar tendon is associated with pain in athletes with patellar tendinopathy', European Journal of Applied Physiology 117(5), 1039-1045.

Leporace, G. Pereira, G.R., Carmo, R.C.R., Silva, A.C., Cabral, R.P., Silva, N. et al., 2010 'Specificity of the myoelectrical activity on the eccentric decline squat at 25 degrees and standard squat with different overloads', Revista Brasileira de degrees and standard squat with
Medicina do Esporte 16(3), 205-209.

Malliaras, P., Barton, C.J., Reeves, N.D. \& Langberg, H., 2013, 'Achilles and patellar tendinopathy loading programmes: A systematic review comparing clinical outcomes and identifying potential mechanisms for effectiveness', Sports Medicine 43(4), 267-286.

Malliaras, P. Cook J. Purdam, C. \& Rio, E, 2015, 'Patellar tendinopathy: Clinica diagnosis, load management, and advice for challenging case presentations', Journal of Orthopaedic and Sports Physical Therapy 45(11), 887-898.

Mani-Babu, S., Morrissey, D., Waugh, C., Screen, H. \& Barton, C., 2015, 'The effectiveness of extracorporeal shock wave therapy in lower limb tendinopathy: A systematic review', American Journal of Sports Medicine 43(3), 752-761.

Mccreesh, K.M., Riley, S.J. \& Crotty, J.M., 2013, 'Neovascularity in patellar tendinopathy and the response to eccentric training: A case report using Power Doppler ultrasound', Manual Therapy 18(6), 602-605.

Mendonça, L.D., Bittencourt, N.F.N., Santos, T.R.T., Silva, A.A. \& Fonseca, S.T., 2011 Correlation of age, sex, body mass index and sports modality to patellar rotation in jumping athletes', British Journal of Sports Medicine 45(4), 344

Morton S., Morrissey D., Valle X., Chan O., Langberg H. \& Malliaras P., 2015 'Equivalence of online and clinical administration of a patella tendinopathy risk factor and severity questionnaire', Scandinavian Journal of Medicine \& Science in Sports 25(5), 670-677.

Morton, S., Williams, S., Valle, X., Diaz-Cueli, D., Malliaras, P. \& Morrissey, D., 2017 'Patellar tendinopathy and potential risk factors: An international database of cases and controls', Clinical Journal of Sport Medicine 27(5), 468-474.

Rio, E., Kidgell, D., Purdam, C., Gaida, J., Moseley, G.L., Pearce, A.J. \& Cook, J., 2015 'Isometric exercise induces analgesia and reduces inhibition in patellar tendinopathy', British Journal of Sports Medicine 49(19), 1277-1283.

Romero-Rodriguez, D., Gual, G. \& Tesch, P.A., 2011, 'Efficacy of an inertial resistance training paradigm in the treatment of patellar tendinopathy in athletes: A caseseries study', Physical Therapy in Sport 12(1), 43-48.

Saggini, R., Di Stefano, A., Galati, V., Panelli, E., Valeri, M., Di Pancrazio, L. et al., 2012 'Long-term effectiveness of combined mechanotransduction treatment in jumper's knee', European Journal of Inflammation 10(3), 515-524.

Samukawa, M., 2011, 'Management of patellar tendinosis in a freestyle mogul skier', International Journal of Athletic Therapy and Training 16(2), 12-15.

Scattone, Silva, R., Nakagawa, T.H., Ferreira, A.L.G., Garcia, L.C., Santos, J.E.M. \& Serrão, F.V., 2016, 'Lower limb strength and flexibility in athletes with and without patellar tendinopathy', Physical Therapy in Sport 20, 19.

Silva, R.S., Ferreira, A.L.G., Nakagawa, T.H., Santos, J.M. \& Serrão, F.V., 2015, 'Rehabilitation of patellar tendinopathy using hip extensor strengthening and landing-strategy modification: Case report with 6-month follow-up', Journal of Orthopaedic and Sports Physical Therapy 45(11), 899-909.

Simpson, M. \& Smith, T.O., 2011, 'Quadriceps tendinopathy - A forgotten pathology for physiotherapists? A systematic review of the current evidence-base', Physical Therapy Reviews 16(6), 455-461.

Sorenson, S.C., Arya, S., Souza, R.B., Pollard, C.D., Salem, G.J. \& Kulig, K., 2010, 'Knee extensor dynamics in the volleyball approach jump: The influence of patellar tendinopathy', Journal of Orthopaedic \& Sports Physical Therapy 40(9), 568-576. 
Souza, R.B., Arya, S., Pollard, C.D., Salem, G. \& Kulig, K., 2010, 'Patellar tendinopathy alters the distribution of lower extremity net joint movements during hopping', Journal of Applied Biomechanics 26(3), 249-255.

Thijs, K.M., Backx, F.J.G., Moen, M.H., Zwerver, J., Steeneken, V., Rayer, S. et al., 2017, 'Effectiveness of shockwave treatment combined with eccentric training for patellar tendinopathy: A double-blinded randomized study', Clinical Journal of Sport Medicine 27(2), 89-96.

Van Der Worp, H., Zwerver, J., Van Den Akker-Scheek, I. \& Diercks, R.L., 2011c, 'The TOPSHOCK study: Effectiveness of radial shockwave therapy compared to focused shockwave therapy for treating patellar tendinopathy - Design of a randomised controlled trail', BMC Musculoskeletal Disorders 12, 229-234.

Wilgen, C.P., Konopka, K.H., Keizer, D. \& Zwerver, J., 2013, 'Do patients with chronic patellar tendinopathy have an altered somatosensory profile? A quantitative sensory testin (QST) study', Scandinavian Journal of Medicine \& Science in Sports 23(2), 149-155.

Yun, S., Jin, W., Park, Y.K., Kim, G., Yoon, S., Park, S. et al., 2015, 'Increased signal intensity at the proximal patellar tendon: Correlation between MR imaging and histology in eight cadavers and clinical MR imaging studies', European Radiology 25(10), 2976-2983.

Zhang, Z., Ng, G., FU, S., Zhang, Z.J., Ng, G.Y.F. \& Fu, S.N., 2015, 'Effects of habitual loading on patellar tendon mechanical and morphological properties in basketball and volleyball players', European Journal of Applied Physiology 115(11), 2263-2269.

Zhang, Z.J., Ng, G.Y., Lee, W.C. \& Fu, S.N., 2014, 'Changes in morphological and elastic properties of patellar tendon in athletes with unilateral patellar tendinopathy and their relationships with pain and functional disability', PLoS One 9(10), 1-9.

Zwerver, J., Dekker, F. \& Pepping, G.J., 2010, 'Patient guided Piezo-electric Extracorporeal Shockwave Therapy as treatment for chronic severe patellar tendinopathy: A pilo study', Journal of Back and Musculoskeletal Rehabilitation 23(3), 111-115.

\section{Exclusion of articles because of poor methodological quality $(n=7)$}

Frizziero, A., Vittadini, F., Fusco, A., Giombini, A. \& Masiero, S., 2016, 'Efficacy of eccentric exercise in lower limb tendinopathies in athletes', The Journal of Sports Medicine and Physical Fitness 56(11), 1352-1358.

Malliaras, P., Cook, J., Purdam, C. \& Rio, E. (2015). Patellar Tendinopathy: Clinical Diagnosis, Load Management, and Advice for Challenging Case Presentations. Journal of Orthopaedic \& Sports Physical Therapy 45(11), 887-898.
Nagraba, L., Mitek, T., Stolarczyk, A., Lebiediew, A. \& Przymorski, T., 2010, 'Tendinopathy of the patellar ligament (jumper's knee) - Diagnostics and treatment', Arthroscopy and Joint Surgery 6(3-4), 19-23.

Pearson, S.J. \& Hussain, S.R., 2014, 'Region-specific tendon properties and patellar tendinopathy: A wider understanding', Sports Medicine 44(8), 1101-1112.

Rodriguez-Merchan, E.C., 2013, 'The treatment of patellar tendinopathy', Journal of Orthopaedic Traumatology 14, 77-81.

Rudavsky, A. \& Cook, J., 2014, 'Physiotherapy management of patellar tendinopathy (jumper's knee)', Journal of Physiotherapy 60(3), 122-129.

Rutland, M., O'Connell, D., Brismee, J.M., Sizer, P., Apte, G. \& O'Connell, J., 2010 'Evidence-supported rehabilitation of patellar tendinopathy', North American Journal of Sports Physical Therapy 5(3), 166-178.

\section{Methodology quality scoring of the individual articles}

\section{Systematic review}

\begin{tabular}{lc}
\hline Author & AMSTAR Checklist Scoring \\
\hline Van Der Worp et al. (2011b) & $8 / 11$ \\
\hline
\end{tabular}

\section{Quantitative research}

\begin{tabular}{lc}
\hline Author & $\begin{array}{c}\text { National Institute for Health and } \\
\text { Excellence Checklist Scoring }\end{array}$ \\
\hline De Vries et al. (2015a) & $21 / 27$ \\
Mendonça et al. (2016) & $18 / 27$ \\
Toppi et al. (2015) & $16 / 27$ \\
Van der Worp et al. (2011a) & $16 / 27$ \\
Van der Worp et al. (2012) & $15 / 27$ \\
\hline
\end{tabular}

